\title{
A Pilot Study: No Therapeutic Effect of L-Alanine in Patients with Nonalcoholic Steatohepatitis
}

\author{
Ayako Suzuki ${ }^{1,2}$, Michael R. Charlton ${ }^{1}$, James F. Lymp ${ }^{3}$, Roberta A. Jorgensen ${ }^{1}$, Jill Keach ${ }^{1}$, Jan Petz ${ }^{1}$, \\ Paul Angulo ${ }^{1}$, Keith Lindor ${ }^{1}$ \\ ${ }^{1}$ Gastroenterology and Hepatology, Mayo Clinic, Rochester, USA; ${ }^{2}$ Gastroenterology, Duke University, Durham, USA; ${ }^{3}$ Seattle \\ Children's Research Institute, Seattle, USA. \\ Email: srivastava.dfrl@gmail.com
}

Received August $4^{\text {th }}, 2010$; revised August 27 $7^{\text {th }}, 2010$; accepted October $9^{\text {th }}, 2010$.

\begin{abstract}
Background: Mitochondrial dysfunction plays a pivotal role in the progression of nonalcoholic steatohepatitis (NASH). L-alanine was shown to restore ATP content and protect the liver in various liver injury models. Aim: To assess the safety and therapeutic effects of long-term administration of L-alanine in patients with NASH, we conducted a pilot trial. Methods: Patients with NASH were enrolled and treated with 6 - $18 \mathrm{~g} /$ day L-alanine for 12 months and monitored for serum aminotransferases and renal function. Liver histology was obtained at baseline and 12 months. Changes in serum aminotransferase were assessed by differences from entry and rate of change per month using all available measures. Changes in liver histology were assessed by differences in Brunt scores of steatosis, lobular inflammation, and fibrosis. Results: Nine patients were enrolled and six completed the treatment. The reasons of the study withdrawal were nausea $(\mathrm{n}=1)$, planned bariatric surgery $(\mathrm{n}=1)$, and un-specified reason $(\mathrm{n}=1)$. One participant experienced exacerbation of pre-existing renal failure that required hospitalization, although the medication was safely resumed after 2-week cessation and treatment was completed. Serum alanine aminotransferase (ALT) $(-24.8 \pm 32.1$ IU/L vs. $0, p$ $=0.11)$ and aspartate aminotransferase $(A S T)(-8 \pm 16.21 \mathrm{IU} / \mathrm{L}$ vs. $0, p=0.28)$ were improved in 4 and 3 of the 6 completed participants, while rate of ALT and AST change per month showed improvement over time (negative slope) in 5 and 4 of the 6. Liver histology did not change significantly. Conclusion: The 12-month administration of L-alanine seems to be safe, but did not show significant therapeutic effects on serum aminotransferase or liver histology in patients with NASH, along with less than ideal tolerability.
\end{abstract}

Keywords: Nonalcoholic Steatohepatitis, Treatment, Hepatic ATP

\section{Introduction}

Nonalcoholic fatty liver disease (NAFLD) has dramatically increased in prevalence and become one of the most common liver diseases in industrialized nations [1,2]. NAFLD includes a broad spectrum of clinico-pathological entities from simple steatosis to steatohepatitis, and cirrhosis. Nonalcoholic steatohepatitis (NASH) is one stage of NAFLD complicated with necroinflammation either with or without fibrosis. A certain portion of patients with NASH may progress to liver cirrhosis, develop hepatocellular carcinoma, or suffer a liver death [1]. Currently, no established treatments for NASH exist [3]. Therefore, effective, safe, and tolerable treatments to prevent the progression of NASH to cirrhosis are needed.

Oxidative stress is thought to play a pivotal role in
NASH progression [4]. The intracellular sources of reactive oxygen species (ROS) includes various enzymes such as cytochrome P450 enzymes (e.g., cytochrome P [CYP] 2E1), NADPH oxidase, lipoxygenases, cyclooxygenases, and xanthine oxidase in addition to mitochondria. Among them, mitochondria are considered as a major source of ROS, particularly in the cells that have high concentrations of mitochondria such as heart and liver [5]. Generation of ROS occur through physiological mitochondrial oxidative phosphorylation; however, it enormously increases under mitochondrial abnormalities [5]. Functional and morphological mitochondrial abnormalities and depressed expression of functional proteins in mitochondria observed in the NASH liver suggests that oxidative stress is one of the promising therapeutic target in patients with NASH [6-8].

Previous studies showed that obese subjects, especially 
subjects with NASH have impaired hepatic ATP homeostasis. Hepatic recovery following hepatic ATP depletion induced by fructose infusion become progressively less efficient as body mass index (BMI) increased and was severely impaired in patients with NASH in a study using nuclear magnetic resonance spectroscopy [9]. Since ATP supplementation is crucial for cellular functions to maintain cell viability as well as recover from injury, the impaired hepatic ATP homeostasis in NASH may also contribute the NASH progression.

L-alanine is a glycogenic amino acid used as a substrate of ATP synthesis. A series of previous animal studies showed potential therapeutic effects of L-alanine in various murine models of liver injury, i.e., D-gal-induced acute liver failure, carbon tetrachloride-induced liver injury in rats, ethanol and hydrazine-induced chronic liver failure models [10-12]. The administration of L-alanine significantly increased ATP content in the liver following ATP depletion induced by D-gal when compared with control groups. L-alanine administration also enhanced gene expression related to mitochondrial function, such as subunits of F0/F1 ATPase, cytochrome C oxidase and some components of the tricarboxylic acid (TCA) cycle [11]. Furthermore, recent studies suggested that the protective effects of $\mathrm{L}$-alanine are related to the restoration of ATP content [13].

To date, there are limited data from human studies [14]. However, the evidence from animal studies provided the hypothesis that the long-term administration of L-alanine may improve necroinflammation and fibrosis in patients with NASH through the augmentation of ATP and regulation of energy metabolism. Based on the rationale described above, we conducted a pilot clinical trial to assess the therapeutic efficacy as well as the safety of Lalanine supplementation in patients with NASH.

Specific aims were to assess in patients with histologically confirmed NASH 1) the safety and tolerability of long-term L-alanine supplementation and 2) the therapeutic efficacy of long-term L-alanine supplementation by evaluating liver biochemistry and histological findings.

\section{Methods}

Study design: This study was an open-label, single-center clinical trial conducted at Mayo Clinic, Rochester, MN. Participants received the study medication for 12 months and were followed as detailed below. The trial was approved by the Institutional Review Boards at Mayo Clinic, Rochester, MN.

Participants: Patients with histologically confirmed NASH who met entry criteria were enrolled in this study. Steatohepatitis was defined as steatosis with moderate to severe hepatocellular injury indicated by ballooning de- generation and diffuse mixed lobular inflammation with or without perisinusoidal and perivenular fibrosis, according to the Brunt classification[15]. Inclusion criteria were: 1) age between 18 and 75,2 ) histological diagnosis of NASH made within one year of entry, 3) alcohol consumption of less than $20 \mathrm{~g} /$ day, and 4) transaminase levels [alanine aminotransferase (ALT) or aspartate aminotransferase (AST)] more than 1.5 times the upper normal limit on at least two occasions with one assessment at three months or more prior to treatment of this study. The following patients were excluded from this study: patients who had 1) any causes for liver disease other than NASH, 2) use of drugs causing steatosis-associated liver diseases within past 6 months (e.g., steroids, tamoxifen, amiodarone, and methotrexate), 3) decompensated liver disease, 4) any previous experimental treatment of NASH within the past 3 months, 5) pre-existing diseases/situations that could interfere with the results or the completion of this trial (e.g., uncontrolled diabetes mellitus, renal or liver transplant patients, and severe cardiovascular dysfunction), and 5) pregnant women. The study was conducted in compliance with the Declaration of Helsinki and approved by appropriate regulatory bodies. All patients gave written informed consent for participation.

Study medication: The therapeutic efficacy and safety of $18 \mathrm{~g} /$ day of L-alanine (equivalent to $0.3 \mathrm{~g} / \mathrm{kg} / \mathrm{day}$ ) (Ajinomoto USA, Fort Lee, NJ) was evaluated in this trial. To ensure safety, the medication was started with a dosage of $6 \mathrm{~g}$ per day (equivalent amount of daily intake of L-alanine from foods in persons consuming $100 \mathrm{~g}$ of protein per day), then gradually increased to $18 \mathrm{~g}$ /day. More specifically, participants took $6 \mathrm{~g}$ of L-alanine (powder) once per day for the first month, twice per day (12 g/day) for the second month, then three times per day (18 g/day) from the third month for 10 months.

Baseline evaluation and monitoring: At enrollment, a complete medical history, physical examination, and laboratory assessment including serum liver biochemistries, fasting blood glucose, and lipid profile were performed. During the study period, adverse reactions and compliance were checked through phone contact at 1,2 , 3, 6, 9, and 12 months. Patients were also asked to record any symptoms, concomitant medication use, compliance, lifestyle changes, and body weight on the provided diary during the study. The participants were examined for liver histology and anthropometric measures at baseline and 12 months and serum liver enzymes [i.e., ALT and AST] at baseline, 1, 2, 3, 6, 9, and 12 months. Renal function and electrolytes (blood and urine) were also assessed at baseline, 1, 2, 3, and 12 months to monitor for safety.

Liver histology: The histological findings at baseline 
and final evaluation were reviewed by a pathologist blinded to the patient's identity as well as to sequence of biopsy. We evaluated the following histologic features of NAFLD in this study: the grades of steatosis, lobular inflammation, and the stage of fibrosis according to Brunt criteria. [15] Briefly, steatosis was graded into 0 to 3 based on the percentage of affected hepatocytes: $<5 \%$ (grade 0), 5-33\% (grade 1), 34-66\% (grade 2) and $>66 \%$ (grade 3). Lobular inflammation was graded into 0 to 3 based on the numbers of inflammatory foci per $20 \times$ field: 0 (grade 0$),<2$ (grade 1), 2-4 (grade 2), and > 4 (grade 3 ). Fibrosis was classified into 5 stages: none, normal connective tissue (stage 0 ), zone 3 perisinusoidal or periportal fibrosis (stage $1 ; 1 \mathrm{a}=$ mild, zone 3 , perisinusoidal, $1 \mathrm{~b}=$ moderate, zone 3 , perisinusoidal, $1 \mathrm{c}=$ portal $/$ periportal), moderate, zone 3, perisinusoidal and portal/ periportal fibrosis (stage 2), bridging fibrosis (stage 3), cirrhosis (stage 4). For the analyses, fibrosis stage 1a, 1b, and 1c were combined and treated as stage 1 .

Statistical analysis: Safety of the study medication was assessed based on the proportion of patients who developed adverse reactions (e.g. symptoms, signs, illnesses, and accidents) during the trial as well as the severity of the reactions. The efficacy of the treatment was assessed by 1) changes in serum liver enzymes (ALT and AST) and 2) changes in histologic grades or stage. Changes in serum liver enzymes were calculated as differences from entry, rate of change per month in serum liver enzymes using all available measures, and as proportion of patients with normalization (and improvement) at 12 months. ALT and AST normalization were defined using the reference ranges used at Mayo Clinic (ALT: $\leq$ $55 \mathrm{IU} / \mathrm{L}$ for males and $\leq 45 \mathrm{IU} / \mathrm{L}$ for females; AST: $\leq 48$ $\mathrm{IU} / \mathrm{L}$ for males and $\leq 43 \mathrm{IU} / \mathrm{L}$ for females). Improvement of each histological finding (steatosis, lobular inflammation, and fibrosis) was defined as at least one level of improvement at 12 months. Confidence intervals for proportions were calculated using the Wilson score method. The study was designed to have $80 \%$ power to detect changes equal to 1 standard deviation (SD) in AST or ALT change with a sample size of 8 . With 6 subjects, there was $80 \%$ power to detect a change equal to $1.15 \mathrm{SD}$. Statistical analyses was conducted using JMP statistical software version 7.0 (SAS Institute Inc.), R version 2.8.1 [16], and Stata version 10.1 (StataCorp, College Station, TX).

\section{Results}

\subsection{Study Accrual and Patient's Characteristics}

A total of 11 patients were enrolled in this trial. Out of the 11 patients, two decided not to participate before the study; therefore, 9 patients received the study medication.
Out of the 9 patients, 3 patients were withdrawn at 5, 6, and 11 months without completing the one-year medication for the following reasons: receiving bariatric surgery and being bothered by too many urine tests $(\mathrm{n}=1)$, symptom (i.e., nausea) due to the medication $(\mathrm{n}=1)$, and unspecified reasons $(\mathrm{n}=1)$.

Baseline clinical characteristics of the study population $(\mathrm{n}=9)$ are summarized in Tables 1(a) and 1(b). Briefly, mean age with standard deviation was $57.4 \pm 9.3$ years old. Of the 9 subjects, 4 were male. Three had diabetes mellitus (stable, well-controlled), 4 had hypertension, and 4 had hyperlipidemia. Two patients had cirrhosis (stage 4).

Of the 6 patients who completed the study, 4 lost weight during the study period. Mean weight changes and $\%$ weight change at 12 months with standard deviation were $-2.8 \pm 5.6 \mathrm{~kg}$ and $-1.9 \pm 4.7 \%$, respectively.

\subsection{Study Medication Compliance}

Compliance information was available in 4 out of the 6 patients who completed one-year of medication: $100 \%$ compliance in two, $>98 \%$ in one, and 2 week cessation in one due to hospitalization (pre-existing renal dysfunction). The information was not available in the other 2 patients.

\subsection{Safety Evaluations}

There were three adverse incidence reports from 3 individuals in this study $(33.3 \%, 95 \% \mathrm{CI}: 12.1 \%-64.6 \%)$ : diarrhea in one patient who completed the study medication, nausea in another patient who requested withdrawal and renal dysfunction in conjunction with pre-existing renal disease in yet another patient who did complete the study medication after 2 -week drug cessation. Otherwise, there were no reported gastrointestinal distress or adverse changes in renal function test [blood urea nitrogen (BUN), creatinine, and creatinine clearance].

\subsection{Efficacy Evaluations}

Changes in serum ALT and AST of all 9 enrolled patients are shown in Figure 1 and Table 2. Of the 6 patients who completed the study, 5 (83\%, 95\% CI: 44\%$97 \%$ ) showed improvement in serum ALT, and $4(67 \%$, 95\% CI: 30\%-90\%) showed improvement in serum AST. ALT (or AST) normalization was observed in $3(50 \%$, 95\% CI: 19\%-81\%) patients. For serum ALT, mean rate of change was $-1.7(\mathrm{sd}=3.0) \mathrm{IU} / \mathrm{L} /$ month $(\mathrm{p}=0.20)$. For serum AST, mean rate of change was $-1.5(\mathrm{sd}=5.5)$ $\mathrm{IU} / \mathrm{month}(\mathrm{p}=0.30)$. The observed changes in serum aminotransferases were not correlated with degrees of weight changes during the study period (data are not shown).

Histological evaluation revealed that out of 6 subjects, 
Table 1. (a) Patient's characteristics at baseline.

\begin{tabular}{|c|c|c|}
\hline & Enrolled Subjects $(\mathrm{N}=9)$ & Subjects Completing $(\mathrm{N}=6)$ \\
\hline Age, years old & $57.4 \pm 9.3$ & $61.7 \pm 3.0$ \\
\hline Gender, Male & $4(44 \%)$ & $3(50 \%)$ \\
\hline BMI, $\mathrm{kg} / \mathrm{m}^{2}$ & $34.3 \pm 6.1$ & $34.0 \pm 2.7$ \\
\hline Diabetes Mellitus* & $3(33 \%)$ & $1(17 \%)$ \\
\hline Hypertension & $4(44 \%)$ & $2(33 \%)$ \\
\hline Hyperlipidemia & $4(44 \%)$ & $3(50 \%)$ \\
\hline ALT, IU/L & $102.9 \pm 66.6$ & $93.8 \pm 28.5$ \\
\hline AST, IU/L & $74.3 \pm 36.0$ & $61.2 \pm 13.1$ \\
\hline Total bilirubin, $\mathrm{mg} / \mathrm{dl}$ & $0.9 \pm 0.6$ & $0.9 \pm 0.3$ \\
\hline Albumin, $\mathrm{g} / \mathrm{dl}$ & $4.2 \pm 0.5$ & $4.2 \pm 0.2$ \\
\hline Platelet count, $\times 10^{9} / \mathrm{L}$ & $207.8 \pm 79.2$ & $233.7 \pm 30.1$ \\
\hline Triglycerides, mg/dl & $193.5 \pm 101.8$ & $199.5 \pm 44.6$ \\
\hline Total. cholesterol, mg/dl & $180.8 \pm 61.5$ & $187.2 \pm 26.6$ \\
\hline HDL-cholesterol, mg/dl & $42.0 \pm 9.7$ & $43.2 \pm 4.2$ \\
\hline LDL-cholesterol, mg/dl & $100.1 \pm 59.9$ & $104.3 \pm 26.2$ \\
\hline Fasting blood sugar, $\mathrm{mg} / \mathrm{dl}$ & $108.7 \pm 26.1$ & $113.2 \pm 11.0$ \\
\hline $\mathrm{Cr}, \mathrm{mg} / \mathrm{dl}$ & $1 \pm 0$ & $1 \pm 0$ \\
\hline $\mathrm{BUN}, \mathrm{mg} / \mathrm{dl}$ & $15.6 \pm 6.2$ & $19.0 \pm 1.5$ \\
\hline $\mathrm{CCr}, \mathrm{ml} / \mathrm{min}$ & $90.7 \pm 20.1$ & $92.8 \pm 10.9$ \\
\hline \multicolumn{3}{|l|}{ Histology } \\
\hline Steatosis, grade & $1.4 \pm 0.7$ & $1.3 \pm 0.3$ \\
\hline Lobular inflammation, grade & $1.2 \pm 0.4$ & $1.2 \pm 0.2$ \\
\hline Fibrosis, stage** & $2.5 \pm 1.2$ & $2.2 \pm 0.5$ \\
\hline
\end{tabular}

*: Stable, well-controlled; **: There is one subject missing baseline fibrosis.

Table 1. (b) Characteristics of individual participants.

\begin{tabular}{|c|c|c|c|c|c|c|c|c|c|}
\hline & \multirow{2}{*}{ Age } & \multirow{2}{*}{ Sex } & \multirow{2}{*}{ DM } & \multirow{2}{*}{ HTN } & \multirow{2}{*}{ HL } & \multicolumn{3}{|c|}{ Baseline histology } & \multirow{2}{*}{ Withdrawn } \\
\hline & & & & & & STE & LOB & FIB & \\
\hline Case 1 & 54.8 & M & No & No & Yes & 1 & 1 & 4 & No \\
\hline Case 2 & 58.6 & $\mathrm{~F}$ & Yes & No & Yes & 1 & 1 & 2 & No \\
\hline Case 3 & 63.8 & $\mathrm{~F}$ & No & No & No & 2 & 2 & 3 & No \\
\hline Case 4 & 54.7 & M & No & Yes & No & 1 & 1 & 1 & No \\
\hline Case 5 & 40.0 & $\mathrm{~F}$ & No & No & No & 3 & 1 & 2 & $11 \mathrm{Mo}$ \\
\hline Case 6 & 71.6 & M & No & No & No & 2 & 1 & - & No \\
\hline Case 7 & 50.2 & $\mathrm{~F}$ & Yes & Yes & Yes & 1 & 2 & 3 & $6 \mathrm{Mo}$ \\
\hline Case 8 & 66.5 & $\mathrm{~F}$ & Yes & Yes & Yes & 1 & 1 & 1 & No \\
\hline Case 9 & 56.5 & M & No & Yes & No & 1 & 1 & 4 & $5 \mathrm{Mo}$ \\
\hline
\end{tabular}

DM: diabetes mellitus; HTN: hypertension; HL: hyperlipidemia; STE: steatosis grade; LOB: lobular inflammation grade; FIB: fibrosis stage; There is one subject missing baseline fibrosis stage. 
Table 2. Changes in serum ALT and AST during the administration of therapeutic dose of study medication.

\begin{tabular}{cccccccc}
\hline & Baseline fibrosis & \multicolumn{3}{c}{ ALT changes } & \multicolumn{3}{c}{ AST changes } \\
\cline { 2 - 8 } & & At entry & $\begin{array}{c}\text { Change at } \\
\text { 12 months }\end{array}$ & $\begin{array}{c}\text { Rate of change } \\
\text { per month }\end{array}$ & At entry & $\begin{array}{c}\text { Change at } \\
\text { 12 months }\end{array}$ & $\begin{array}{c}\text { Rate of change } \\
\text { per month }\end{array}$ \\
\hline Case 1 & 4 & 180 & 7 & 1.414 & 112 & 11 & 1.009 \\
Case 2 & 2 & 26 & 0 & -1.158 & 34 & 0 & -0.694 \\
Case 3 & 3 & 67 & -8 & -0.794 & 48 & 6 & 0.272 \\
Case 4 & 1 & 154 & -80 & -6.840 & 82 & -30 & -2.724 \\
Case 5 & 2 & 222 & - & -1.500 & 112 & - & -10.500 \\
Case 6 & - & 81 & -38 & -1.911 & 55 & -21 & -0.949 \\
Case 7 & 3 & 89 & - & -5.491 & 130 & - & -7.575 \\
Case 8 & 1 & 55 & -30 & -3.386 & 36 & -14 & -1.609 \\
Case 9 & 4 & 52 & - & 1.000 & 60 & - & 9.000 \\
Mean & & 102.9 & -24.8 & -1.7 & 74.3 & -8.0 & -1.5 \\
SD & & 66.6 & 32.1 & 3.0 & 36.0 & 16.2 & 5.5 \\
N & & -6 & 6 & 9 & 9 & 6 & 9 \\
P-value* & & - & 0.11 & 0.20 & - & 0.28 & 0.30 \\
\hline
\end{tabular}

* P-values based on two-sided Wilcoxon rank sum test (vs. 0); Case 5, 7, and 9 were withdrawn at 11,6 , and 5 months, respectively.

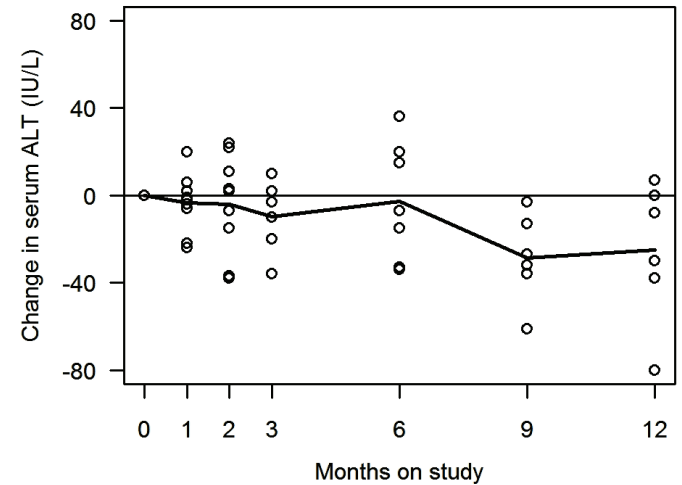

(a)

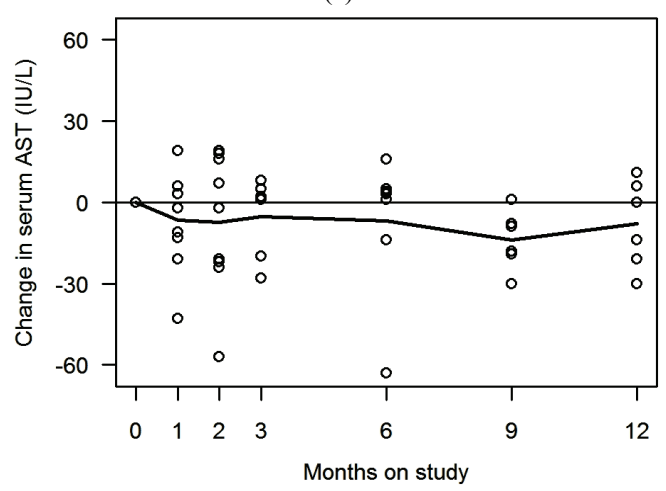

(b)

Figure 1. Serum ALT and AST changes during the study. Changes in serum alanine aminotransferase (ALT) and aspartate aminotransferase (AST) during the study are shown in Figure 1(a) and 1(b) respectively. Horizontal axis is time as months, and vertical axis is serum ALT (or AST) levels $(\mathrm{IU} / \mathrm{L})$. Each open circle in the figures represents data from individuals. Solid line represents mean values at each time point. Figures depict the changes in all the 9 participants, including 3 subjects who were withdrawn without completing the study.

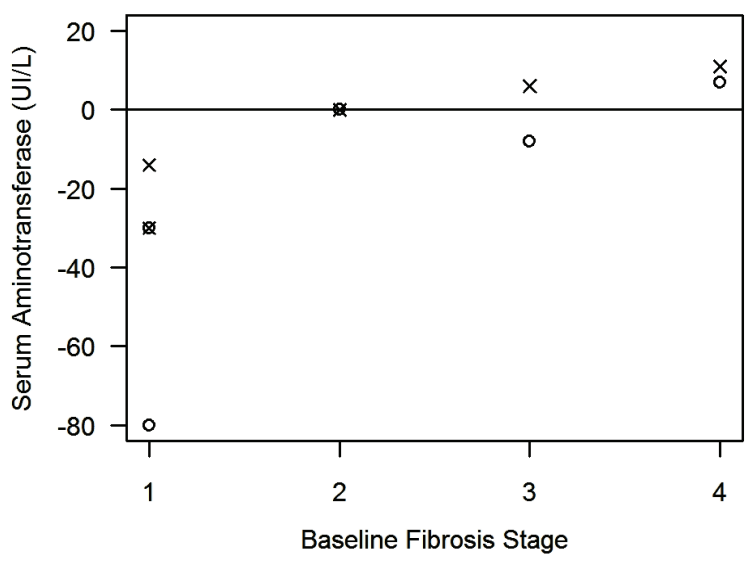

Figure 2. The associations between baseline fibrosis stages and changes of serum aminotransferase at 12 months. Figure presents the associations of baseline fibrosis stages (stage 1-4) (horizontal axis) with changes of serum alanine aminotransferase (ALT) at 12 months (IU/L) (O) and aspartate aminotransferase (AST) at 12 months (IU/L) (×) (vertical axis). The milder fibrosis at baseline was associated with greater improvement (negative value) in serum aminotransferase, while advanced fibrosis (stage 3-4) was associated with less improvement or deterioration (positive values) in serum aminotransferase.

one $(17 \%, 95 \% \mathrm{CI}: 3 \%-56 \%)$ showed improvement in steatosis, one showed improvement in lobular inflamma-tion, but zero (0\%, 95\% CI: 0\%-39\%) showed improvement in fibrosis.

\subsection{Secondary Analysis}

We further analyzed associations of the response of ALT (or AST) to the study medication with age, gender, body weight changes, baseline serum aminotransferase values, 
and baseline histology. As shown in Figure 2, there was a positive correlation between baseline fibrosis stage and aminotransferase change at 12 months; milder stages at baseline were associated with a greater improvement (greater negative values), while severe stages at baseline were associated with less improvement or deterioration (small negative values to a positive value). This correlation still existed when using rate of aminotransferase changes per month instead (data are not shown). No associations were observed with age, gender, body weight changes during the study period, baseline steatosis or lobular inflammation.

\section{Discussion}

In this study, we conducted a pilot study to evaluate safety and efficacy of L-alanine in patients with histologically proven NASH. The one-year treatment with L-alanine, which was preclinically shown to restore ATP content in the liver, was acceptably safe. However, currently available data in this trial did not provide hard evidence supporting the efficacy of L-alanine in patients with NASH nor ideal tolerability. The summary statistics of serum aminotransferases showed that 4 to 5 out of 6 patients had a trend of improvement (negative slope), except for patients with advanced fibrosis. Further, the observed improvement in aminotransferases was not associated with weight loss. Thus, at this point, we cannot conclude whether there is no effect of alanine or that the insignificant results were due to the low power of this small sample to detect a beneficial effect of alanine.

Further, the study population in this study had mild inflammation at baseline; mean lobular inflammation grade was 1.2. Based on our recently published data, estimated probability of spontaneous improvement in hepatic inflammation in NASH patients was $3.0 \%$ for grade 1 and $17.8 \%$ for grade 2 , and $60 \%$ for grade 3 [17], as compared with $16.7 \%$ of improvement in lobular inflammation in this study. Thus, proportion of histologic improvement observed in this study should be interpreted with caution, taking into account baseline histologic grades or stage.

Next, our secondary analysis shows that aminotransferase changes (aminotransferase response to the administration of alanine) were influenced by baseline fibrosis stage. This relationship still existed even after taking account of baseline serum aminotransferase levels (data are not shown). This relationship is opposite to "the regression to the mean", which has been observed in a historical cohort of patients with nonalcoholic steatohepatitis with two-year follow-up [17], suggesting that this association might be specifically associated with the L-alanine treatment. It would be conceivable that alanine might be effective only in patients without advanced fibrosis. However, this study cannot provide conclusive explanation for this association. Further exploration is required to delineate mechanisms explaining this possible stage-specific effect. This study has limitations. A small sample size with considerable drop-out limited our ability to assess the therapeutic effect of L-alanine with sufficient statistical power. We used liver histology as one of the study outcomes; inter-observer variance [18] and sampling error $[19,20]$ may have affected our findings, which need to be considered in the data interpretation. Use of newly available measures (such as cytokeratin-18 fragment [21], transient elastography [22]) or use of biological or physiological endpoints (such as intra-hepatic oxidative stress, hepatic ATP homeostasis measured by nuclear magnetic resonance spectroscopy [9]) may be future consideration for assessing the effect of L-alanine.

In summary, the one-year treatment of L-alanine (up to $18 \mathrm{~g} /$ day) in patients with NASH was acceptably safe. This study, however, did not show significant therapeutic effects on aminotransferases or liver histology nor ideal tolerability. Considering the sampling variability, together with the above-discussed points, whether L-alanine is beneficial on patients with non-advanced NASH is still an open question. Further exploration with reconsideration of dosage and formula may be warranted.

\section{Acknowledgements}

The authors would like to thank Dr. Fumihiko Takatsuki (Ajinomoto Co., Inc., Kawasaki, Japan) for his expertise and supports on this trial.

\section{REFERENCES}

[1] L. A. Adams and K. D. Lindor, "Nonalcoholic Fatty Liver Disease," Annals of Epidemiology, Vol. 17, No. 1, 2007, pp. 863-869.

[2] S. Kojima, et al., "Increase in the Prevalence of Fatty Liver in Japan over the Past 12 Years: Analysis of Clinical Background," Journal of Gastroenterology, Vol. 38, No. 10, 2003, pp. 954-961.

[3] L. A. Adams and P. Angulo, "Treatment of Non-Alcoholic Fatty Liver Disease," Postgraduate Medical Journal, Vol. 82, No. 967, 2006, pp. 315-322.

[4] A. J. Sanyal, "Mechanisms of Disease: Pathogenesis of Nonalcoholic Fatty Liver Disease," Nature Reviews Gastroenterology \& Hepatology, Vol. 2, No. 1, 2005, pp. 4653.

[5] P. Jezek and L. Hlavata, "Mitochondria in Homeostasis of Reactive Oxygen Species in Cell, Tissues, and Organism," International Journal of Biochemistry \& Cell Biology, Vol. 37, No. 12, pp. 2478-2503.

[6] H. Kojima, et al., "Mitochondrial Abnormality and Oxidative Stress in Nonalcoholic Steatohepatitis, Alcohol," Clinical and Experimental Research, Vol. 31, No. (Suppl. 
1), 2007, pp. S61-S66.

[7] E. Santamaria, et al., "Functional Proteomics of Nonalcoholic Steatohepatitis: Mitochondrial Proteins as Targets of S-Adenosylmethionine," Proceedings of the National Academy of Sciences of the United States of America, Vol. 100, No. 6, 2003, pp. 3065-3070.

[8] A. J. Sanyal, et al., "Nonalcoholic Steatohepatitis: Association of Insulin Resistance and Mitochondrial Abnormalities," Gastroenterology, Vol. 120, No. 5, 2001, pp. 1183-1192.

[9] H. Cortez-Pinto, et al., "Alterations in Liver ATP Homeostasis in Human Nonalcoholic Steatohepatitis: A Pilot Study," Jama, Vol. 282, No. 17, 1999, pp. 1659-1664.

[10] K. Maezono, et al., "Alanine Protects Liver from Injury Caused By F-Galactosamine and CCl4," Hepatology, Vol. 24, No. 1, 1996, pp. 185-191.

[11] K. Maezono, et al., "Effect of Alanine on D-Galactosamine-Induced Acute Liver Failure in Rats," Hepatology, Vol. 24, No. 5, 1996, pp. 1211-1216.

[12] H. Suzuki, et al., "Ethanol and Hydrazine Sulfate Induced Chronic Hepatic Injury in Rats: The Curative Effect of Administration of Glucogenic Amino Acids," Alcohol Alcohol, No. Suppl.1A, 1993, pp. 111-117.

[13] S. Ishizaki-Koizumi, et al., "Mechanisms of the Protective Effect of L-Alanine to D-Galactosamine-Induced Hepatocellular Injury: Comparative Studies of L-Alanine and Pyruvate," Biochemical and Biophysical Research and Communication, Vol. 291, No. 4, 2002, pp. 738-743.

[14] S. Nishiguchi, "Effects of Alanine in Patientswith Advanced Primary Biliary Cirrhosis: Preliminary Report,"
Hepatology Research, 2002.

[15] E. M. Brunt, et al., "Nonalcoholic Steatohepatitis: A Proposal for Grading and Staging the Histological Lesions," American Journal of Gastroenterology, Vol. 94, No. 9, 1999, pp. 2467-2474.

[16] R Development Core Team, R: A Language and Environment for Statistical Computing, 2010, R Foundation for Statistical Computing, Vienna, Austria. http://www. R-project.org

[17] A. Suzuki, et al., "Values and Limitations of Serum Aminotransferases in Clinical Trials of Nonalcoholic Steatohepatitis," Liver International, Vol. 26, No. 10, 2006, pp. 1209-1216.

[18] D. E. Kleiner, et al., "Design and Validation of a Histological Scoring System for Nonalcoholic Fatty Liver Disease," Hepatology, Vol. 41, No. 6, 2005, pp. 1313-1321.

[19] V. Ratziu, et al., "Sampling Variability of Liver Biopsy in Nonalcoholic Fatty Liver Disease," Gastroenterology, Vol. 128, No. 7, 2005, pp. 1898-1906.

[20] A. Regev, et al., "Sampling Error and Intraobserver Variation in Liver Biopsy in Patients with Chronic HCV Infection," American Journal of Gastroenterology, Vol. 97, No. 10, 2002, pp. 2614-2618.

[21] A. E. Feldstein, et al., "Cytokeratin-18 Fragment Levels as Noninvasive Biomarkers for Nonalcoholic Steatohepatitis: A Multicenter Validation Study," Hepatology, Vol. 50, No. 4, 2009, pp. 1072-1078.

[22] J. Vergniol, et al., "Changes of Non-Invasive Markers and FibroScan Values during HCV Treatment," Journal of Viral Hepatitis, Vol. 16, No. 2, 2009, pp. 132-140. 